\title{
Leaning Tower illusion: Orientation contrast or perspective distortion? Reply to Maniatis
}

Ali Yoonessi, Elena Gheorghiu, Frederick A A Kingdom

McGill Vision Research, Department of Ophthalmology, McGill University, 687 Pine Avenue W., Montreal H3A 1A1, Quebec, Canada; e-mail: ali.yoonessi@mcgill.ca

Received 26 September 2008, in revised form 1 October 2008

Maniatis suggests that the Leaning Tower illusion (figure 1a) is not an illusion of perspective, as proposed by us (Kingdom et al 2007a, 2007b), but a variant of the Jastrow illusion. Maniatis suggests that the Jastrow illusion is an example of simultaneous size contrast, and that the Leaning Tower illusion is the orientation analog of the Jastrow. In other words the Leaning Tower illusion is an example of simultaneous tilt (or orientation) contrast, or acute-angle expansion, in which acute angles appear greater than they actually are.

In fact, this idea was first suggested, and rejected, by Akiyoshi Kitaoka in the news section of his illusion website (http://www.psy.ritsumei.ac.jp/akitaoka/illnews5e.html). Kitaoka's line of reasoning, which we reported in Kingdom et al (2007b), was that the acuteangle expansion explanation would predict a similar-sized illusion in figure $1 \mathrm{~b}$, where the arrangement of the inner side of the two child figures is similar to that of the Pisa towers, but in which the figures do not appear to recede. If the illusion was due to acute-angle expansion, one should obtain a similar-sized illusion as with the Pisa towers, whereas if the perspective explanation is correct one should not observe the illusion. The illusion in Kitaoka's figure is tiny or non-existent.

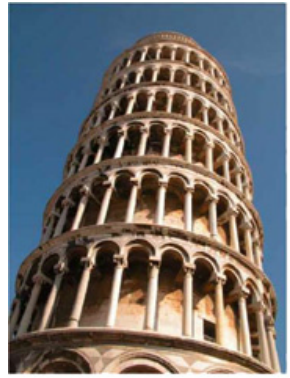

(a)

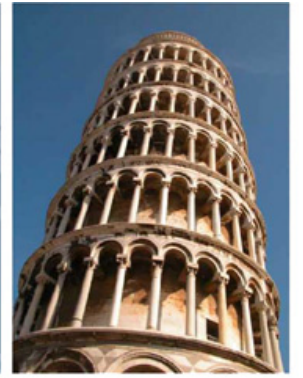

Figure 1. [In colour online, see http://dx.doi.org/10.1068/p6282.] (a) The Leaning Tower illusion. (b) Kitaoka's figure with similar angle relations but devoid of a sense of perspective showing no comparable illusion (reproduced with permission).

Maniatis suggests that a schematic representation of the Leaning Tower illusion, reproduced here in figure $2 \mathrm{a}$ (left), produces the same illusion even though there is little impression of perspective. Unfortunately the persons to whom we have shown this figure report that the illusion is either very weak or non-existent. Maniatis correctly points out that the addition of horizontal lines increases the size of the illusion (figure $2 \mathrm{a}$, right). But don't these lines increase the sense of perspective? And how would this be explained by acute-angle expansion? Figure 2b, another figure adapted from Kitaoka's website, further reinforces the perspective explanation. The addition of curved lines, which 

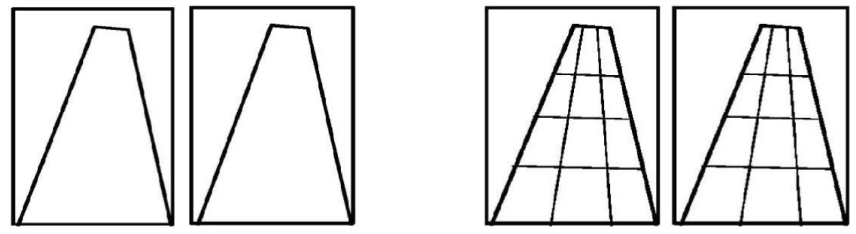

(a)
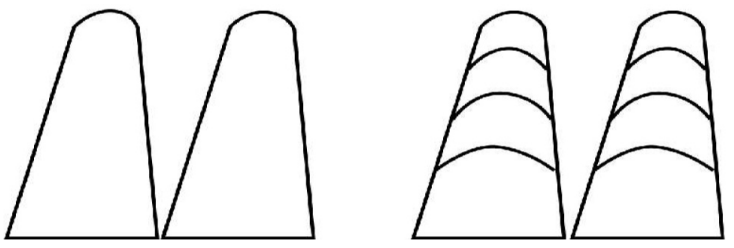

(b)

Figure 2. (a) Schematic representation of the Pisa towers, as suggested by Maniatis, without (left) and with (right) internal contours. (b) Another schematic representation of the Pisa towers, this time without (left) and with (right) curved contours. The right hand figure in (b) is adapted from a similar figure on Kitaoka's website.

strongly promote a three-dimensional (and hence perspective) interpretation, significantly increases the size of the illusion.

Maniatis argues that our perspective explanation implies that when parallel lines are used to define objects that appear to recede in a flat image the lines should always appear to diverge. She provides two counter-examples, reproduced here in figure 3a. First, we fail to perceive the trapezoids in the left figure as receding, so Maniatis's argument does in our view apply to this figure. Her more interesting counter-example is the single, elongated cube on the right, which does appear to recede, yet whose parallel lines do not appear to diverge. Figure $3 \mathrm{~b}$ (left), which is based on a similar figure in Kingdom et al (2007b), suggests, however, that when texture is added to the
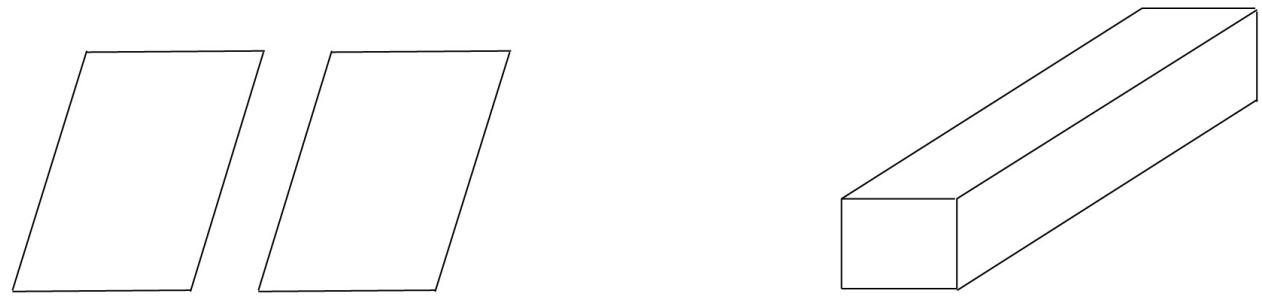

(a)
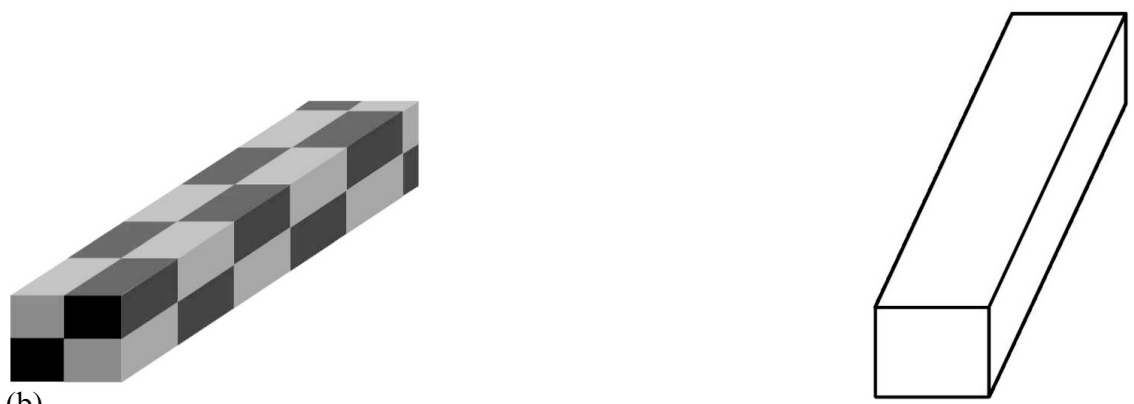

(b)

Figure 3. (a) Left: Maniatis's trapezoids do not, for us, appear to recede. Right: Maniatis's elongated cube contains parallel lines and appears to recede but not diverge. (b) Left: Maniatis's elongated cube with added texture. Right: An elongated cube drawn from a different viewing angle appears to diverge. 
figure the elongated cube does appear to expand with distance. Presumably the addition of texture both reduces the depth-ambiguity inherent in Maniatis's figure, where prolonged fixation can cause depth reversals, and also strengthens the sense of perspective. Whether or not surface texture is necessary to produce the apparent divergence, however, the acute-angle expansion explanation cannot explain the divergence when it does occur, whereas perspective can. The angles in Maniatis's elongated cube might also constitute a special case, as the figure on the right of figure $3 \mathrm{~b}$, which does appear to diverge, suggests.

Maniatis makes a final point: "The original tower is both rotated clockwise within the frontal plane and effectively tilted back, away from the frontal plane .... The relative tilts of the adjacent edges detached from the frontal plane might arguably be responsible for the illusion. Given that each tower seems tilted back, it would then make perspective sense that the right-hand tower should be seen as having more backward tilt than the left" (our emphasis). To us this reads as an argument for the perspective explanation of the illusion.

\section{References}

Kingdom F A A, Yoonessi A, Gheorghiu E, 2007a "The Leaning Tower illusion: a new illusion of perspective" Perception $36475-477$

Kingdom F A A, Yoonessi A, Gheorghiu E, 2007b "Leaning Tower illusion" Scholarpedia 2 5392; http://scholarpedia.org/article/Leaning_Tower_Illusion 


\section{PERTEPTION}

VOLUME 372008

www.perceptionweb.com

Conditions of use. This article may be downloaded from the Perception website for personal research by members of subscribing organisations. Authors are entitled to distribute their own article (in printed form or by e-mail) to up to 50 people. This PDF may not be placed on any website (or other online distribution system) without permission of the publisher. 